ESAIM: COCV 21 (2015) 1108-1119

DOI: $10.1051 / \mathrm{cocv} / 2014060$
ESAIM: Control, Optimisation and Calculus of Variations

www.esaim-cocv.org

\title{
PLANAR INFINITE-HORIZON OPTIMAL CONTROL PROBLEMS WITH WEIGHTED AVERAGE COST AND AVERAGED CONSTRAINTS, APPLIED TO CHEEGER SETS
}

\author{
IDO BRIGHT ${ }^{1}$
}

\begin{abstract}
We establish a Poincaré-Bendixson type result for a weighted averaged infinite horizon problem in the plane, with and without averaged constraints. For the unconstrained problem, we establish the existence of a periodic optimal solution, and for the constrained problem, we establish the existence of an optimal solution that alternates cyclicly between a finite number of periodic curves, depending on the number of constraints. Applications of these results are presented to the shape optimization problems of the Cheeger set and the generalized Cheeger set, and also to a singular limit of the one-dimensional Cahn-Hilliard equation.
\end{abstract}

Mathematics Subject Classification. 49J15, 49N20, 49Q10.

Received November 2, 2013. Revised November 10, 2014

Published online 24 June 2015.

\section{INTRODUCTION}

The celebrated Poincaré-Bendixson theorem was originally stated for smooth planar ordinary differential equations, but since has been extended to flows, semi-flows and differential inclusions. (See, Ciesielski $[10,11]$ for a review on the Poincaré-Bendixson theorem and its generalizations). In the context of infinite horizon optimization, Poincaré-Bendixson type results were obtained for second order Lagrangian by Leizarowitz and Mizel [17], for control systems by Artstein and Bright [2], and for discounted infinite-horizon problems by Colonius and Sieveking [12] and in Bright [6].

In this paper we extend the infinite horizon result to a wider class of values that include weighted average optimization, with an averaged constraint. Two motivations for studying this problem are: The planar shape optimization problem of the Cheeger set and its generalization; and the van der Waals-Cahn-Hilliard theory of phase transition $[7,19]$.

A planar Cheeger set in a domain $\Omega \subset \mathbb{R}^{2}$ is a set $E \subset \Omega$, that maximizes the ratio between its area and the length of its boundary. One over the maximal ratio is the Cheeger constant of the problem. The Cheeger constant is used to bound the first eigenvalue of the Laplacian (see [9]), and generalized Cheeger sets appear in the study of landslides (see [15]). Average constraints arise in the study of phase transition, where the steady states of a partial differential equations is the minimizer of an energy functional with a conserved quantity (in this case

Keywords and phrases. Infinite-horizon optimization, periodic optimization, averaged constraint, planar cheeger set, singular limit, occupational measures, Poincaré-Bendixson.

1 Department of Applied Mathematics, University of Washington, Seattle, WA 98195, United States. ibright@uw.edu 
the conservation of mass), and when a singular limit is present, qualitative properties can be studied through the solution of an infinite horizon problem with an averaged constraint.

The structure of this paper is as follows. The following section contains definitions used throughout the paper, the standing assumptions and some lemmas and previous results used in the proof of the main result. In Section 3 the main results are presented. In Section 4, we present applications of the main result; we establish the reduction of the planar Cheeger problem and its generalization to infinite horizon optimization, and we apply our result to the singular limit of the Cahn-Hilliard equation, a constrained optimization problem. The last section verifies the main results.

\section{Notations, ASSUMPTIONS AND LEMMAS}

\subsection{General notations and assumptions}

The following notations are used throughout the paper. We denote the set of Reals by $\mathbb{R}$, vectors in the Euclidean space $\mathbb{R}^{2}$ by $x=\left(x^{1}, x^{2}\right)$ and $y=\left(y^{1}, y^{2}\right)$, and the standard Euclidean norm by $|\cdot|$. Given a metric space $X$, we denote its probability space by $P(X)$. The action of a measure $\mu \in P(X)$ on a continuos function $g(\cdot) \in C(X)$ is denote by $\mu(g)=\int_{X} g(x) \mu(\mathrm{d} x)$, and given a set of measures $\mathcal{S} \subset P(X)$ and a function $g(\cdot)=\left(g^{1}(\cdot), \ldots, g^{m}(\cdot)\right) \in C\left(X, \mathbb{R}^{m}\right)$ we denote the set $\{\mu(g) \mid \mu \in \mathcal{S}\} \subset \mathbb{R}^{m}$ as its realization by $g(\cdot)$.

Throughout the paper we assume that the control system

$$
\frac{\mathrm{d} x}{\mathrm{~d} t}=f(x, u)
$$

satisfies the following conditions: The function $f(x, t)$ is continuos, and it satisfies Lipschitz conditions in $x$. The constraint set $K \subset \mathbb{R}^{2}$ is compact, and so is the control set $U \subset \mathbb{R}^{d}$. We consider the optimization only with respect to solutions, with measurable controls satisfying $u(t) \in U$, for almost every $t \geq 0$, defined on $[0, \infty)$ and satisfying $x(t) \in K$ for every $t \geq 0$. A solution satisfying the latter conditions is denoted as feasible. We assume that there exists at least one feasible curve.

To obtain the convexity of the set of limiting measures (see Def. 2.3), we require the following controllability assumption, that holds, for example, when for every $x_{0} \in K$ the convex hull of $\left\{f\left(x_{0}, u\right) \mid u \in U\right\} \subset \mathbb{R}^{2}$ contains an open ball around the origin.

Definition 2.1. The control system (2.1) is uniformly controllable in $K \subset \mathbb{R}^{2}$, if there exists a $T_{K}>0$, such that it is possible to steer between any two points $x_{1}, x_{2} \in K$ in time less than $T_{K}$, while staying in the constraint set $K$.

\subsection{Relaxed controls}

A relaxed controls, say $\nu(\cdot)$, is a Young measure (see [22]), where for each time point $\nu(t) \in P(U)$. With relaxed controls the dynamics turn to $f(x, \nu)=\int_{U} f(x, u) \nu(\mathrm{d} u)$ and the cost follows an equivalently representation.

Relaxed controls are introduced in this problem by limits of feasible curves. Indeed, any sequence of solutions of (2.1) defined in a common bounded interval, contains a subsequence converging, in the sense of Young measures, to a solution of (2.1), perhaps, with relaxed controls.

The main result establishes an optimal periodic or stationary solution with relaxed controls. It is well known that in any bounded interval, any solution of the relaxed system can be approximated using only regular controls (see [21], Chap. IV), and in ([2], Sect. 7) the approximation on an infinite time domain is discussed.

\subsection{Occupational measures}

We consider the probability space $P(K \times U)$ endowed with the topology of weak convergence, where $\mu_{i} \rightarrow \mu$ weakly if $\lim _{i \rightarrow \infty} \mu_{i}(g)=\mu(g)$ for every $g \in C(K \times U)$. By our assumptions $K \times U$ is compact which implies 
that the set $P(K \times U)$ is weakly compact, namely, every sequence contains a converging subsequence converging to a measure in $P(K \times U)$. (See [4], Chap. 1)

Definition 2.2. We define the occupational measure $\mu \in P(K \times U)$ corresponding to the curve $(x(\cdot), u(\cdot))$ defined on $[0, T]$, by

$$
\mu(A)=\frac{1}{T} \lambda(t \in[0, t] \mid(x(t), u(t)) \in A),
$$

for every Borel set $A \subset K \times U$, where $\lambda$ is the Lebesgue measure. Notice, that for every $g \in C(K \times U)$

$$
\mu(g)=\int_{K \times U} g(x, u) \mu(\mathrm{d} x, \mathrm{~d} u)=\frac{1}{T} \int_{0}^{T} g(x(t), u(t)) \mathrm{d} t .
$$

Definition 2.3. We denote $\mu$ as a limiting occupational measure if there exists a feasible solution $(x(\cdot), u(\cdot))$ of (2.1), and a sequence $T_{i} \rightarrow \infty$ such that the sequence of occupational measures corresponding to the restriction of $(x(\cdot), u(\cdot))$ to the intervals $\left[0, T_{i}\right]$ converges weakly to $\mu$. The set of all limiting occupational measures is denoted by $\mathcal{M} \subset P(K \times U)$.

With an additional controllability assumption a simple diagonalization argument implies that $\mathcal{M}$ satisfies the following property.

Proposition 2.4. If system (2.1) is uniformly controllable (see Def. 2.1) then $\mathcal{M}$ is convex and closed in the weak topology.

We now state a result from ([2], Lem. 8.2) on the set of occupational measures.

Theorem 2.5. Suppose $g: K \times U \rightarrow \mathbb{R}^{n}$ is continuous. Let

$$
\Delta=\left\{\mu(g)=\int_{K \times U} g(x, u) \mu(\mathrm{d} x, \mathrm{~d} u) \mid \mu \in \mathcal{M}\right\} \subset \mathbb{R}^{n},
$$

be a realization of the set of limiting occupational measures. Every extreme point of the (not necessarily convex) set $\Delta$ corresponds to a stationary solution or a periodic solution with image being a Jordan curve. Namely, given an extreme point $y \in \Delta$, there exists a feasible pair $\left(x_{p}(\cdot), u_{p}(\cdot)\right)$ defined on $\left[0, T_{p}\right]$, possibly, with relaxed controls, with corresponding measure $\mu_{p}$ such that $x(0)=x\left(T_{p}\right)$ and

$$
y=\mu_{p}(g)=\int_{K \times U} g(x, u) \mu_{p}(\mathrm{~d} x, \mathrm{~d} u)=\frac{1}{T_{p}} \int_{0}^{T} g\left(x_{p}(t), u_{p}(t)\right) \mathrm{d} t .
$$

\subsection{Convexity lemmas}

We use the following lemmas.

Lemma 2.6. Suppose $\Delta \subset \mathbb{R}^{n}$ is a convex set and $\Pi \subset \mathbb{R}^{n}$ is an affine subspace of dimension $n-d$. If the set $\Pi \cap \Delta$ is non-empty then each of its extreme points can be represented as a convex combination of $d+1$ extreme points of $\Delta$.

Proof. By Caratheodory's theorem, every point in $\Delta$ is a convex combination of $n+1$ of its extreme points. If $y \in \Pi \cap \Delta$ can be expressed as a convex combination of $k>d+1$ distinct extreme points of $\Delta$ with non-zero coefficients, then, locally, $\Delta$ contains a convex set of dimension $k-1>d$ containing $y$ in its relative interior. The intersection of the latter set with $\Pi$ contains a line centered at $y$, thus $y$ is not an extreme point of $\Pi \cap \Delta$.

Corollary 2.7. Suppose $\Delta \subset \mathbb{R}^{n}$ is a convex set and $\Pi_{1}, \ldots, \Pi_{m} \subset \mathbb{R}^{n}$ are affine subspaces of dimension $n-d$. If the set $\Delta \cap \Pi_{1} \cap \cdots \cap \Pi_{m}$ is non-empty then each of its extreme points can be represented as a convex combination of $d * m+1$ extreme points of $\Delta$. 


\section{MAin RESUlts}

Our main result establishes a Poincaré-Bendixson type results for a wide class of averaged infinite-horizon optimization problems. The main problem we consider is the optimization of the ratio of integrals. We present our main results for this value, but they hold for a wider class of optimization problems. (See Rem. 3.7 and Thm. 5.1.).

We wish to minimize (or maximize, see, Rem. 3.6)

$$
v^{*}=\limsup _{T \rightarrow \infty} \frac{\int_{0}^{T} p(x(t), u(t)) \mathrm{d} t}{\int_{0}^{T} q(x(t), u(t)) \mathrm{d} t},
$$

with respect to all feasible solutions of equation (2.1).

Two type of constraints are considered:

(1) Cumulative constraint: Given a single continuous function $C_{1}(x, u)$, we consider only feasible solutions satisfying

$$
\int_{0}^{T} C_{1}(x(t), u(t)) \mathrm{d} t \leq 0, \quad \forall T \geq 0
$$

(2) Averaged constraints: Given $m \geq 1$ continuous functions $C_{1}(x, u), \ldots, C_{m}(x, u)$ we consider only feasible solutions which for every $k=1, \ldots, m$ satisfy either

$$
\limsup _{T \rightarrow \infty} \frac{1}{T} \int_{0}^{T} C_{k}(x(t), u(t)) \mathrm{d} t \leq 0
$$

or

$$
\lim _{T \rightarrow \infty} \frac{1}{T} \int_{0}^{T} C_{k}(x(t), u(t)) \mathrm{d} t=0 .
$$

In this paper, as is the general case in infinite horizon problems with average costs, we do not consider an initial condition. This is due to the fact that the behavior of the solution in any finite time domain has no effect on the total value of the problem.

For the unconstrained problem, we extend a previous result in [2], establishing the existence of a periodic optimal solution. For the constrained optimization problem, given by $m$ constraints, we establish the existence of a solution that is either stationary or periodic, or alternates between, at most, $m+1$ such solutions, in a cyclic manner.

We now state our main results.

Theorem 3.1. Suppose $p(x, u)$ and $q(x, u)$ are continuous, and $q(x, u)$ is positive. The minimization of

$$
v^{*}=\limsup _{T \rightarrow \infty} \frac{\int_{0}^{T} p(x(t), u(t)) \mathrm{d} t}{\int_{0}^{T} q(x(t), u(t)) \mathrm{d} t}
$$

restricted to feasible solutions of (2.1) is attained by a stationary solution, or by a periodic solution with image being a Jordan curve. The solution may use relaxed control (see Sect. 2.2).

Theorem 3.2. Suppose system (2.1) is uniformly controllable, $p(x, u), q(x, u), C_{1}(x, u), \ldots, C_{m}(x, u)$ are continuous, and $q(x, u)$ is positive. Consider the minimization of (3.3) restricted to feasible solutions of (2.1), satisfying $m$ averaged constraints of the form (3.2a) or (3.2b). If there exists at least one feasible solution satisfying all the constraints, then the minimum is attained by a curve that alternates cyclicly between $m+1$ solutions of (2.1), each of which is either a periodic solution with image being a Jordan curve, or a stationary solution. The solution may use relaxed control.

Moreover, when $m=1$, a stronger constraint of the form (3.1) can be attained in a similar manner. 
Relaxed controls are needed to obtain the desired periodic or stationary optimal solution, as can be seen in the following example taken from ([2], example 7.1).

Example 3.3. Consider the scalar optimization problem given by $f(x, u)=u, p(x, u)=x^{2}+(|u|-1)^{2}$ and $q(x, u) \equiv 1$, which represents the minimization of

$$
v^{*}=\limsup _{T \rightarrow \infty} \frac{1}{T} \int_{0}^{T}\left[(x(t))^{2}+\left(\left|\frac{\mathrm{d} x}{\mathrm{~d} t}(t)\right|-1\right)^{2}\right] \mathrm{d} t .
$$

The value of this problem is $v^{*}=0$, it can be attained by a zigzag curve having derivatives in $\{+1,-1\}$ almost everywhere, with a amplitude converging to 0 . It is clear that using regular control the value of every periodic solution is larger than 0 . But using a relaxed control equidistributed in $\{+1,-1\}$, one achieves an optimal stationary solution.

Remark 3.4. While the periodic or stationary optimal solution provided by Theorems 3.1 and 3.2 may be achieved only through relaxed controls, it can be approximated with regular controls, in the same manner discussed in [2].

Remark 3.5. The condition that $q(x, t)$ is positive can be relaxed by requiring that its integral over every periodic curve is uniformly bounded from below by a positive number.

Remark 3.6. The problem is stated as the minimization of the limit superior, however, since the minimum is attained, we can replace the limit superior by the limit. Also, replacing $p(x, u)$ by $-p(x, u)$ we see that our result generalize to the maximization problems as well.

Remark 3.7. The minimization problem is stated for a weighted average cost, however, it holds (see, Thm. 5.1) for a wider class of problems, such as the minimization of

$$
\inf _{(x(\cdot), u(\cdot))} \limsup _{T \rightarrow \infty} \frac{1}{T}\left(\int_{0}^{T} p_{1}(x(t), u(t)) \mathrm{d} t+\left|\int_{0}^{T} p_{2}(x(t), u(t)) \mathrm{d} t\right|\right) .
$$

\section{Motivations And application: Shape optimization And Partial Differential EQUATIONS}

In this section, we present an application of our result to the planar shape optimization problem of the Cheeger set, and show how qualitative properties of the singular limit of the Cahn-Hilliard equation can be obtained based on the corresponding infinite horizon optimization problem with an averaged constraint.

\subsection{Shape optimization: Cheeger sets and generalized Cheeger sets}

The planar isoperimetric problem is the first known shape optimization problem, where one seeks, amongst all sets $E \subset \mathbb{R}^{2}$ with a given perimeter, a set with maximal area. This problem was reformulated to sets that maximize the ratio between their area and their perimeter, in a given bounded domain $\Omega \subset \mathbb{R}^{2}$. We denote the maximal ratio by

$$
v_{C}^{*}=\max _{E \subset \bar{\Omega}} V(E)=\max _{E \subset \bar{\Omega}} \frac{\operatorname{Area}(E)}{\operatorname{Length}(\partial E)} .
$$

The Cheeger constant is then defined by $1 / v_{C}^{*}$. It is well-known that the maximum might not be unique, and that it is attained by a set with boundary being a Jordan curve. The set maximizing (4.1) is called a Cheeger set, which we denote by

$$
E^{*}=\arg \max _{E \subset \bar{\Omega}} \frac{\operatorname{Area}(E)}{\operatorname{Length}(\partial E)}, \partial E^{*} \text { is a rectifiable Jordan curve. }
$$


The Cheeger constant appear in the context of partial differential equation. The Cheeger inequality bounds from below the largest eigenvalue of the laplacian equation with homogenous boundary conditions by $\frac{1}{4}\left(v_{C}^{*}\right)^{-2}[9]$. Cheeger sets are difficult to compute, and only recently Kawohl and Lachand-Robert [16] provide an analytic characterization for convex planar domains.

Cheeger sets have been generalized to generalized Cheeger sets, which arise in applications in landslide modeling [15]. These sets maximize

$$
v_{G C}^{*}=\max _{E \subset \Omega} V_{P ; Q}(E)=\max _{E \subset \Omega} \frac{\int_{E} P(x) \mathcal{L}^{n}(\mathrm{~d} x)}{\int_{\partial^{*} E} Q(x) \mathcal{H}^{n-1}(\mathrm{~d} x)},
$$

where $P(\cdot)$ and $Q(\cdot)$ are continuous, $\mathcal{L}^{n}$ denotes the $n$-dimensional Lebesgues measure, and $\mathcal{H}^{n-1}$ denotes the $n$-1-dimensional Hausdorff measure. Similar to the Cheeger set problem, the averaging property implies that the optimal value is attained by a set with boundary being a rectifiable Jordan curve.

The reduction to infinite-horizon optimization allows the use of tools from infinite horizon optimization to solve these shape optimization problems. In particular, numerical computation of the infinite horizon problem in Proposition 4.1 was performed to estimate the Cheeger set and Cheeger constant, where they can be computed analytically in order that the numerical result can be evaluated, using semi-infinite linear programing (see [13] and [14]). Two examples were computed: The $6 \mathrm{x} 4$ rectangle and the ovoid domain $\left(x^{2}+y^{2}\right)^{2}<x^{3}$. In both cases the Cheeger constant was computed with accuracy of 4 digits, and for the rectangle the computed solution was within $2 \times 10^{-3}$ from the boundary of the theoretic Cheeger set.

In order to formulate problems (4.1) and (4.3) as infinite horizon control problems, we first express them by an integral over a parametrized curve.

Let $x(\cdot)=\left(x^{1}(\cdot), x^{2}(\cdot)\right)$ be a parametrized Jordan curve defined on $[0, T]$, with interior $E$ and positive orientation. The area of $E$ can be expressed as

$$
\operatorname{Area}(E)=\int_{0}^{T} x^{1}(t) \frac{\mathrm{d} x^{2}}{\mathrm{~d} t}(t) \mathrm{d} t
$$

and the length of $\partial E$ as

$$
\text { Length }(\partial E)=\int_{0}^{T}\left|\frac{\mathrm{d} x}{\mathrm{~d} t}(t)\right| \mathrm{d} t .
$$

The integrals in expression (4.3) can be expressed, when $P(\cdot) \in C(\Omega)$, by

$$
\int_{E} P(x) \mathcal{L}^{n}(\mathrm{~d} x)=\int_{0}^{T} P_{1}(x(t)) \frac{\mathrm{d} x^{2}}{\mathrm{~d} t}(t) \mathrm{d} t,
$$

where

$$
P_{1}\left(\left(x^{1}, x^{2}\right)\right)=\int_{0}^{x^{1}} P\left(\zeta, x^{2}\right) \mathrm{d} \zeta
$$

and

$$
\int_{\partial^{*} E} Q(x) \mathcal{H}^{n-1}(\mathrm{~d} x)=\int_{0}^{T} Q(x(t))\left|\frac{\mathrm{d} x}{\mathrm{~d} t}(t)\right| \mathrm{d} t .
$$

Thus, we have that the ratio between a set's area and perimeter can be expressed as

$$
V(E)=\frac{\operatorname{Area}(E)}{\operatorname{Length}(\partial E)}=\frac{\int_{0}^{T} x^{1}(t) \frac{\mathrm{d} x^{2}}{\mathrm{~d} t}(t) \mathrm{d} t}{\int_{0}^{T}\left|\frac{\mathrm{d} x}{\mathrm{~d} t}(t)\right| \mathrm{d} t},
$$

and the generalized Cheeger set problem can be expressed as

$$
V_{P ; Q}(E)=\frac{\int_{0}^{T} P_{1}(x(t)) \frac{\mathrm{d} x^{2}}{\mathrm{~d} t}(t) \mathrm{d} t}{\int_{0}^{T} Q(x(t))\left|\frac{\mathrm{d} x}{\mathrm{~d} t}(t)\right| \mathrm{d} t} .
$$


To simplify things more, we consider solutions of $(2.1)$, where $f(x, u)=u$ and $U=\partial B(\mathbf{0}, 1)=$ $\left\{y \in \mathbb{R}^{2}|| y \mid=1\right\} \subset \mathbb{R}^{2}$. Then, the latter expressions reduce to

$$
V(E)=\frac{\int_{0}^{T} x^{1}(t) u^{2}(t) \mathrm{d} t}{\int_{0}^{T}|f(x, u)| \mathrm{d} t}=\frac{1}{T} \int_{0}^{T} x^{1}(t) u^{2}(t) \mathrm{d} t,
$$

and

$$
V_{P ; Q}(E)=\frac{\int_{0}^{T} P_{1}(x(t)) u^{2}(t) \mathrm{d} t}{\int_{0}^{T} Q(x(t)) \mathrm{d} t},
$$

where $u(\cdot)=\left(u^{1}(\cdot), u^{2}(\cdot)\right)$.

With these identities established, we apply our main result to obtain the equivalence between the shape optimization problem and the infinite horizon problem.

Proposition 4.1. Suppose the control system (2.1) is defined by the vector field $f(x, u)=u$ with constraint set $K=\bar{\Omega}$ and control set $U=\partial B(\mathbf{0}, 1)=\left\{y \in \mathbb{R}^{2}|| y \mid=1\right\} \subset \mathbb{R}^{2}$. The maximization problem

$$
v^{*}=\sup _{(x(\cdot), u(\cdot))} \limsup _{T \rightarrow \infty} \frac{1}{T} \int_{0}^{T} x^{1}(t) u^{2}(t) \mathrm{d} t
$$

with respect to feasible solutions of (2.1), is attained by a periodic curve with arc-length parametrization, such that its image is a Jordan curve bounding a set $E$, which satisfies $V(E)=v^{*}=v_{G C}^{*}$.

Proof. Theorem 3.1 implies that the value is attained either by a Jordan curve or by a stationary solution. The value of a stationary solution is 0 , on the other hand, any Jordan curve, with positive orientation, has positive value. Thus, the solution is attained by $\left(x^{*}(\cdot), u^{*}(\cdot)\right)$ corresponding to a Jordan curve bounding a set $E_{J}$. The period of this solution satisfies $T^{*} \geq \operatorname{Length}\left(\partial E_{J}\right)$ (the inequality can be strict when relaxed controls are used). Thus, by (4.4), $v^{*}=\operatorname{Area}\left(E_{J}\right) / T^{*} \leq \operatorname{Area}\left(E_{J}\right) /$ Length $\left(\partial E_{J}\right)=V\left(E_{J}\right) \leq v_{C}^{*}$, which implies that $v^{*} \leq v_{C}^{*}$.

We now prove that $v \geq v_{C}^{*}$. Let $E^{*}$ be given by (4.2). Applying (4.6) to a parametrized curve with arc-length parametrization of $\partial E^{*}$ with positive orientation, we conclude that $v^{*} \geq V\left(E^{*}\right)=v_{C}^{*}$, and $v^{*}=V\left(E^{*}\right)=v_{C}^{*}$, which completes the proof.

A Similar result holds for generalized Cheeger sets.

Proposition 4.2. Suppose $P(\cdot), Q(\cdot) \in C(K)$ are positive, and $P_{1}(\cdot)$ is defined by (4.5). Consider the maximization problem

$$
v^{*}=\sup _{(x(\cdot), u(\cdot))} \limsup _{T \rightarrow \infty} \frac{\int_{0}^{T} P_{1}(x(t)) \frac{\mathrm{d} x^{2}}{\mathrm{~d} t}(t) \mathrm{d} t}{\int_{0}^{T} Q(x(t)) \mathrm{d} t},
$$

with respect to feasible solutions of $(2.1)$, given by the vector field $f(x, u)=u$ and the sets $K=\bar{\Omega}$ and $U=\partial B(\mathbf{0}, 1)=\left\{y \in \mathbb{R}^{2}|| y \mid=1\right\} \subset \mathbb{R}^{2}$. The maximum is attained by a periodic curve with arc-length parametrization, such that its image is a Jordan curve bounding a set $E$, which satisfies $v^{*}=V_{P ; Q}(E)=v_{G C}^{*}$.

This result can be generalized to the case when $Q(x, u)$ depends on the control.

Proposition 4.3. Suppose $P(\cdot) \in C(K)$ is positive, $P_{1}(x)$ is defined by (4.5) and the integral of $Q(x, u) \in$ $C(K \times U)$, over every periodic solution, is bounded from below by some $\eta>0$. Consider the maximization problem

$$
v^{*}=\sup _{(x(\cdot), u(\cdot))} \limsup _{T \rightarrow \infty} \frac{\int_{0}^{T} P_{1}(x(t)) \frac{\mathrm{d} x^{2}}{\mathrm{~d} t}(t) \mathrm{d} t}{\int_{0}^{T} Q(x(t), u(t)) \mathrm{d} t},
$$


with respect to feasible solutions of (2.1) given by the vector field $f(x, u)=u$ and the sets $K=\Omega$ and $U=\partial B(\mathbf{0}, 1)=\left\{y \in \mathbb{R}^{2}|| y \mid=1\right\} \subset \mathbb{R}^{2}$. The optimal solution is attained by a Jordan curve. Perhaps, with relaxed controls.

Proof. By our assumption on $Q(x, u)$ the minimization of

$$
v_{Q}^{*}=\limsup _{T \rightarrow \infty} \frac{1}{T} \int_{0}^{T} Q(x(t), u(t)) \mathrm{d} t
$$

is attained by a periodic solution. This implies that $v_{Q}^{*} \geq \eta>0$ and we can apply Theorem 5.1 , and the proof follows the observation that the value of a stationary solution is 0 .

\subsection{Singular limits of partial differential equations}

Constrained optimization appears in the study of singular limits of partial differential equations, where a steady state solution is a minimizer of an energy functional with a constraint. As an example, we study 1-dimensional phase transitions, modeled by the van der Waals-Cahn-Hilliard theory. We show how our main result can be applied to obtain qualitative properties of the singular limit.

The limit of a solution of the Cahn-Hilliard equation

$$
\frac{\mathrm{d}}{\mathrm{d} t} u_{\epsilon}(x, t)=\frac{\mathrm{d}^{2}}{\mathrm{~d} x^{2}}\left(-\epsilon^{2} \frac{\mathrm{d}^{2}}{\mathrm{~d} x^{2}} u_{\epsilon}(x, t)+W\left(u_{\epsilon}(\zeta, \tau)\right)\right), \quad u_{\epsilon}(\cdot, 0)=u_{0}(\cdot), u_{\epsilon}(0, \cdot)=-1, u(1, \cdot)=1,
$$

where $\epsilon$ is a small parameter, is also the minimizer of the van der Waals free energy given by

$$
E_{\epsilon}=\int_{0}^{1}\left(W\left(u_{\epsilon}(x)\right)+\frac{\epsilon^{2}}{2}\left(u_{\epsilon}^{\prime}(x)\right)^{2}\right) \mathrm{d} x, u_{\epsilon}(0)=-1, u_{\epsilon}(1)=1,
$$

where $u_{\epsilon}^{\prime}(x)=\frac{\mathrm{d}}{\mathrm{d} x} u_{\epsilon}(x)$, constrained by the conservation of mass

$$
\int_{0}^{1} u_{\epsilon}(x) \mathrm{d} x=\int_{0}^{1} u_{0}(x) \mathrm{d} x=M .
$$

Note that we use a different parametrization than the one found in the literature for the double-well potential, given by a function satisfying $W \geq 0$ and $W=0$ only at 0 and 1 . There one minimizes

$$
\epsilon^{-1} E_{\epsilon}=\int_{0}^{1}\left(\epsilon^{-1} W\left(u_{\epsilon}(x)\right)+\frac{\epsilon}{2}\left(u_{\epsilon}^{\prime}(x)\right)^{2}\right) \mathrm{d} x .
$$

While our results can only provide information on the general structure of the solution of the singular limit, the latter parametrization provides the exact solution, being a step function, as well as the limit of $\epsilon^{-1} E_{\epsilon}$, which is $2 \sqrt{\int_{0}^{1} W(u) \mathrm{d} u}$ (rather than the trivial limit $E_{\epsilon} \rightarrow 0$, in this case.). The double well potential problem was first solved by Modica and Mortola [18], using $\Gamma$-convergence, and Carr et al. [8] also solved the latter problem with tools from singular perturbations. For additional information on solving singularly perturbed optimization problems using $\Gamma$-convergence, also with application to the Cahn-Hilliard problem, the reader is referred to Alberti [1], Braides [5] and Sternberg [20], and to references within.

Our interest in this problem is in the limit of $u_{\epsilon}(\cdot)$ as $\epsilon$ goes to zero, and in $v^{* *}=\liminf _{\epsilon \rightarrow 0} E_{\epsilon}$. Assuming sufficient (quadratic) growth conditions on $W(\cdot)$ it is easy to see that for all $\epsilon$ small enough, the minimizers are uniformly bounded and uniformly Lipschitz.

By ([2], Prop. 6.1) we can reduce the singular limit problem to the constrained infinite horizon problem minimizing

$$
v^{*}=\limsup _{T \rightarrow \infty} \frac{1}{T} \int_{0}^{T}\left(W(u(\zeta))+\frac{\left(u^{\prime}(\zeta)\right)^{2}}{2}\right) \mathrm{d} \zeta,
$$


with free initial condition and average constraint

$$
\lim _{T \rightarrow \infty} \frac{1}{T} \int_{0}^{T} W(u(\zeta)) \mathrm{d} \zeta=M,
$$

with the same constraint set and Lipschitz constant as in the singular limit. The reduction implies that $v^{*}=v^{* *}$ and that the occupational measure of solutions $u_{\epsilon}(\cdot)$ converge to an occupational measure of an optimal solution of the infinite horizon equation. Note, that ([2], Prop. 6.1) considers the unconstrained case, however, it can easily be extended to the constrained case we are considering.

Artstein and Leizarowitz [3] show that the minimum of unconstrained first order scalar Lagrangians is attained by stationary solution. Thus, according to the methods in Section 5 there is an optimal measure which is a convex combination of two measures corresponding to stationary solutions, which for the double well potential, studied in [8], is in fact unique. Thus, by ([2], Prop. 6.1), the singular limit is concentrated at two points as expected by [8].

The Lagrangian in (4.10) is a first order scalar Lagrangian, for second order Lagrangians, with a single averaged constraint, Theorem 3.2 suggests, the appearance of approximate "piecewise periodic" optimal solutions for small $\epsilon$. Namely, solutions that first approximately follow one periodic solution, with velocity of order $O\left(\epsilon^{-1}\right)$, for a time period of order $O(1)$, and then continues to approximately follow a second periodic solution till $x=1$.

\section{Proof OF MAIN RESUlT}

The existence of an optimal solution to our problem relies on a convexity property of the set of limiting occupational measures $\mathcal{M}$ (see, Def. 2.3). To employ this property, we first restate the optimization problem using occupational measures, reducing (3.3) to

$$
v^{*}=\inf _{\mu \in \mathcal{M}} \mu(p) / \mu(q)=\inf _{\mu \in \mathcal{M}}\left(\int_{K \times U} p(x, u) \mu(\mathrm{d} x, \mathrm{~d} u) / \int_{K \times U} q(x, u) \mu(\mathrm{d} x, \mathrm{~d} u)\right) .
$$

The main theorems follows from the general result below.

Theorem 5.1. Suppose $g(x, u)=\left(g^{m}(x, u), \ldots, g^{n}(x, u)\right) \in C\left(K \times U, \mathbb{R}^{n}\right)$ and

$$
\Delta=\left\{\mu(g)=\int_{K \times U} g(x, u) \mu(\mathrm{d} x, \mathrm{~d} u) \mid \mu \in \mathcal{M}\right\} \subset \mathbb{R}^{n}
$$

be the realization of $\mathcal{M}$ by $g(x, u)$. If $V \in C(\Delta)$ is quasi-concave, namely, for every $z_{1}, z_{2} \in \Delta$

$$
\min \left\{H\left((1-\lambda) z_{1}+\lambda z_{2}\right) \mid 0 \leq \lambda \leq 1\right\}=\min \left\{H\left(z_{1}\right), H\left(z_{2}\right)\right\},
$$

then:

(1) The optimization problem

$$
v^{*}=\inf _{\mu \in \mathcal{M}} V\left(\mu\left(g^{1}\right), \ldots, \mu\left(g^{n}\right)\right),
$$

attains its optimal solution by a measure corresponding to a periodic or stationary solution of (2.1).

(2) If system (2.1) is uniformly controllable (Def. 2.1) then the constrained optimization problem

$$
v^{*}=\inf _{\mu \in \mathcal{M}^{c}} V\left(\mu\left(g^{1}\right), \ldots, \mu\left(g^{n}\right)\right),
$$

where

$$
\mathcal{M}^{C}=\left\{\mu \in \mathcal{M} \mid \mu\left(g^{k}\right) \leq 0 \text { for } k=1, \ldots m\right\} \neq \emptyset,
$$


attains its minimum by a measure $\mu^{*} \in \mathcal{M}^{C}$ such that

(a) $V\left(\mu^{*}\left(g^{1}\right), \ldots, \mu^{*}\left(g^{n}\right)\right)=v^{*}$.

(b) The measure $\mu^{*}$ is a convex combination of $m+1$ occupational measures, corresponding to stationary or periodic solutions of (2.1).

(c) There is a feasible solution, corresponding to the measure $\mu^{*}$, that alternates between the $m+1$ stationary or periodic solutions from (b).

(d) When $m=1$ an optimal solution $\left(x^{*}(\cdot), u^{*}(\cdot)\right)$ of the form $(\mathrm{c})$ exists, such that

$$
\int_{0}^{T} g^{1}\left(x^{*}(t), u^{*}(t)\right) \mathrm{d} t \leq 0
$$

for every $T>0$.

Proof. The function $V(\cdot)$ is quasi-concave, thus, its minimum in $\Delta$ is attained in one of its extreme points and Theorem 2.5 implies the result for the unconstrained case.

For the constrained problem, we observe that the set $\Delta_{C}=\left\{\mu(g) \in \mathbb{R}^{n} \mid \mu \in \mathcal{M}_{C}\right\}$, can be expressed as

$$
\Delta_{C}=\Delta \cap \Pi_{1} \cap \cdots \cap \Pi_{m}
$$

where for every $k=1, \ldots, m$ we define the subspace

$$
\Pi_{k}=\left\{\left(z^{1}, \ldots, z^{n}\right) \in \mathbb{R}^{n} \mid z^{k} \leq 0\right\} .
$$

Uniform controllability implies that two point within a compact set can be steered in a finite time, thus, by Proposition 2.4 the set $\Delta$ is compact and convex, and so is $\Delta_{C}$. The function $V(\cdot)$ is quasi-concave, so it attains its minimum in an extreme point of $\Delta_{C}$, which by Corollary 2.7 can be expressed as a convex combination of $m+1$ extreme points of $\Delta$. This completes the proof of (a) and (b).

Suppose that $\mu^{*}=\sum_{j=1}^{m+1} \lambda_{j} \mu_{j}^{p}$ is the minimizing measure, and that each measure $\mu_{j}^{p}$ corresponds to a periodic or stationary solution $\left(x_{j}^{p}(\cdot), u_{j}^{p}(\cdot)\right)$. The uniform controllability implies that there exists a $T_{K}$ that bounds the time it takes to steer between any two points in $K$. We now construct a solution corresponding to the measure $\mu^{*}$ that alternates between the solutions $\left(x_{j}^{p}(\cdot), u_{j}^{p}(\cdot)\right)$.

We start at $x_{1}^{p}(0)$, the initial point of the first curve, and for $i=1,2, \ldots$, we do the following:

(1) for $j=1, \ldots, m$ follow the $j$ th curve for $i \lambda_{j}$ units the time, then steer, in time $\leq T_{K}$, to $x_{j+1}^{p}(0)$, the initial point of the $(j+1)$ th curve.

(2) Follow the $(m+1)$ th curve for $i \lambda_{m+1}$ units of time, then steer, in time $\leq T_{K}$, back to $x_{1}^{p}(0)$, the initial point of the first curve.

When $m=1$, let us first consider the case when the optimal measure $\mu^{*}$ is attained by a periodic solution. Let $\left(x^{*}(\cdot), u^{*}(\cdot)\right)$ be the corresponding curve and $T^{*}$ its period (if it is stationary we attribute it a period of $\left.T^{*}=1\right)$. Thus,

$$
\mu^{*}\left(g^{1}\right)=\frac{1}{T^{*}} \int_{0}^{T^{*}} g^{1}\left(x^{*}(t), u^{*}(t)\right) \mathrm{d} t \leq 0 .
$$

Setting $\tau$ as the points that maximizes the periodic function

$$
F(s)=\int_{0}^{s}\left(g^{1}\left(x^{*}(t), u^{*}(t)\right)-\mu^{*}\left(g^{1}\right)\right) \mathrm{d} t
$$

and translating, in time, the curve $\left(x^{*}(\cdot), u^{*}(\cdot)\right)$ by $\tau$, assures us that $F(\cdot)$ is non positive, and that the constraint is satisfied. 
Otherwise, the optimal measure is of the form $\mu^{*}=\lambda \mu_{1}+(1-\lambda) \mu_{2}$, where each $\mu_{j} \in \mathcal{M}$ corresponds to a periodic trajectory $\left(x_{j}^{p}(\cdot), u_{j}^{p}(\cdot)\right)$, with period $T_{j}$ (where we attribute stationary solutions with period 1 ). In this case, we can assume that $\mu_{1}\left(g^{1}\right)<0<\mu_{2}\left(g^{1}\right)$.

We assume the first solution satisfies $\int_{0}^{s}\left(g^{1}\left(x_{1}^{p}(t), u_{1}^{p}(t)\right)-\mu_{1}\left(g^{1}\right)\right) \mathrm{d} t \leq 0$ for every $s>0$, applying a time translation if necessary, and that the same condition holds for the second solution as well.

In order to accommodate our previous construction to a non averaged constraint, we take into consideration the transient parts between the two curves. To this end we define $\alpha=2 M_{g} T_{K} /\left(-\mu_{1}\left(g^{1}\right)\right)$, where $M_{g}$ bounds $g^{1}(x, u)$ in $K \times U$.

We start our optimal curve at the point $x_{1}^{p}(0)$, then for $i=1,2, \ldots$ we repeat the following:

(1) Follow the curve $\left(x_{1}^{p}(\cdot), u_{1}^{p}(\cdot)\right)$ for time $T_{1}\left\lceil\frac{\alpha}{T_{1}}\right\rceil+T_{1}\left\lceil\frac{\lambda i}{T_{1}}\right\rceil$ time, where $\lceil\beta\rceil$ denotes the smallest integer larger or equal to $\beta$. (The first term takes into account the transitions between the first and second curves, and is needed to make sure the constraint holds.).

(2) Steers to $x_{2}^{p}(0)$, in time $\leq T_{K}$.

(3) Follow the curve $\left(x_{2}^{p}(\cdot), u_{2}^{p}(\cdot)\right)$ for $T_{2}\left\lceil\frac{(1-\lambda) i}{T_{2}}-1\right\rceil$ time.

(4) Steers, in time $\leq T_{K}$, back to the point $x_{1}^{p}(0)$.

Remark 5.2. The definition of $\mathcal{M}_{C}$ in Theorem 5.1 can be relaxed to include equalities as well as inequalities, setting

$$
\mathcal{M}_{C}=\mathcal{M}_{1} \cap \cdots \cap \mathcal{M}_{m}
$$

where for every $k=1, \ldots, m$ either $\mathcal{M}_{k}=\left\{\mu \in \mathcal{M} \mid \mu\left(g_{k}\right) \leq 0\right\}$ or $\mathcal{M}_{k}=\left\{\mu \in \mathcal{M} \mid \mu\left(g_{k}\right)=0\right\}$.

We now present the proofs of Theorems 3.1 and 3.2.

Proof of Theorem 3.1. Let $g(x, u) \in C\left(K \times U, \mathbb{R}^{2}\right)$ be defined by $g^{1}(x, u)=p(x, u)$ and $g^{2}(x, u)=q(x, u)$. Set $m=0$ and $V\left(\left(z_{1}, z_{2}\right)\right)=z_{1} / z_{2}$. Since $q(x, u)>0$, the function $V(\cdot)$ is quasi-concave in its domain, thus, applying Theorem 5.1 completes the proof.

Proof of Theorem 3.2. Let $g(x, u) \in C\left(K \times U, \mathbb{R}^{2+m}\right)$ be defined by $g^{k}(x, u)=C_{k}(x, u)$ for $k=1, \ldots, m$ and $g^{m+1}(x, u)=p(x, u), g^{m+2}(x, u)=q(x, u)$. Set $n=m+2$ and $V\left(\left(z_{1}, z_{2}, \ldots, z_{m+2}\right)\right)=z_{m+1} / z_{m+2}$. The function $V(\cdot)$ is quasi-concave in its domain, thus, combining Theorem 5.1 and Remark 5.2 completes the proof.

The necessity to alternate between stationary solutions of the constrained optimization problem is depicted in the following scalar examples.

Example 5.3. Suppose the control system (2.1) is defined by $f(x, u)=u$ and $K=U=[1,-1]$. The minimization of

$$
v^{*}=\limsup _{T \rightarrow \infty} \frac{1}{T} \int_{0}^{T}\left(1-x(t)-(x(t))^{2}\right) \mathrm{d} t
$$

with respect to feasible solutions satisfying $\int_{0}^{T} x(t) \mathrm{d} t \leq 0$ for every $T>0$, attains the optimal value $v^{*}=0$ by a solution that alternates between -1 and 1 . This follows from the fact that substituting the constraint in the value, we see that for every $T$

$$
\int_{0}^{T}\left(1-x(t)-(x(t))^{2}\right) \mathrm{d} t=\int_{0}^{T}\left(1-(x(t))^{2}\right) \mathrm{d} t-\int_{0}^{T} x(t) \mathrm{d} t \geq T-\int_{0}^{T}(x(t))^{2} \mathrm{~d} t \geq 0,
$$

which implies $v^{*} \geq 0$. Moreover, one concludes from (5.3) that there is no periodic optimal solution. 
Example 5.4. When the value function $V(\cdot)$ in Theorem 5.1 is not concave, the necessity to alternate between stationary solutions is depicted in the following example. The minimization of

$$
v^{*}=\limsup _{T \rightarrow \infty} \frac{1}{T}\left(\left|\int_{0}^{T} x(t) \mathrm{d} t\right|-\int_{0}^{T}|x(t)| \mathrm{d} t\right),
$$

with respect to the system in Example 5.3, is not attained by a periodic solution. Clearly $v^{*} \geq-1$, and the minimum is attained by a solution alternating between the points +1 and -1 . Notice that the function $V(\cdot)$ is convex and the maximization problem is attained by the stationary solution $x \equiv 0$ (as the triangle inequality implies that $v^{*} \leq 0$ for every feasible solution).

Acknowledgements. The author wishes to thank Sergey Rossomakhine for conducting the numerical computations of the Cheeger set and Cheeger constant.

\section{REFERENCES}

[1] G. Alberti, Variational models for phase transitions, an approach via $\Gamma$-convergence. Calc. Var. Partial Differ. Equ. Edited by G. Buttazzo, A. Marino and M.K.V. Murthy. Springer, Berlin, Heidelberg (2000) 95-114.

[2] Z. Artsein and I. Bright, Periodic optimization suffices for infinite horizon planar optimal control. SIAM J. Control Optim. 48 (2010) 4963-4986.

[3] Z. Artstein and A. Leizarowitz, Singularly perturbed control systems with one-dimensional fast dynamics. SIAM J. Control Optim. 41 (2002) 641-658.

[4] P. Billingsley, Convergence of probability Measures. Wiley, New York (1968).

[5] A. Braides, A handbook of $\Gamma$-convergence. Vol. 3 of Stationary Partial Differential Equations. Edited by M. Chipot and P. Quittner. North-Holland (2006) 101-213.

[6] I. Bright, A reduction of topological infinite-horizon optimization to periodic optimization in a class of compact 2-manifolds. J. Math. Anal. Appl. 394 (2012) 84-101.

[7] J.W. Cahn and J.E. Hilliard, Free energy of a nonuniform system. i. interfacial free energy. J. Chem. Phys. 28 (1958) $258-267$.

[8] J. Carr, M.E. Gurtin and M. Slemrod, Structured phase transitions on a finite interval. Arch. Ration. Mech. Anal. 86 (1984) $317-351$.

[9] J. Cheeger, A lower bound for the smallest eigenvalue of the Laplacian. Probl. Anal. 625 (1970) 195-199.

[10] K. Ciesielski, On the Poincaré-Bendixson theorem. Lect. Notes Nonlin. Anal. 3 (2002) 49-69.

[11] K. Ciesielski, The Poincaré-Bendixson Theorem: from Poincaré to the XXIst century. Cent. Eur. J. Math. 10 (2012) $2110-2128$.

[12] F. Colonius and M. Sieveking, Asymptotic properties of optimal solutions in planar discounted control problems. SIAM J. Control Optim. 27 (1989) 608.

[13] V. Gaitsgory and S. Rossomakhine, Linear programming approach to deterministic long run average problems of optimal control. SIAM J. Control Optim. 44 (2006) 2006-2037.

[14] V. Gaitsgory, S. Rossomakhine and N. Thatcher, Approximate solution of the HJB inequality related to the infinite horizon optimal control problem with discounting. Dynam. of Contin. Discrete Impuls. Syst. Ser. B Appl. Algorithms 19 (2012) 65-92.

[15] I.R. Ionescu and T. Lachand-Robert, Generalized Cheeger sets related to landslides. Calc. Var. Partial Differ. Equ. 23 (2005) 227-249.

[16] B. Kawohl and T. Lachand-Robert, Characterization of Cheeger sets for convex subsets of the plane. Pacific J. Math. 225 (2006) 103-118.

[17] A. Leizarowitz and V.J. Mizel, One dimensional infinite-horizon variational problems arising in continuum mechanics. Arch. Ration. Mech. Anal. 106 (1989) 161-194.

[18] L. Modica and S. Mortola, Un esempio di $\Gamma$-convergenza. Boll. Un. Mat. It. B 14 (1977) 285-299.

[19] J.S. Rowlinson, Translation of J.D. van der Waals: The thermodynamik theory of capillarity under the hypothesis of a continuous variation of density. J. Stat. Phys. 20 (1979) 197-200.

[20] P. Sternberg, The effect of a singular perturbation on nonconvex variational problems. Arch. Ration. Mech. Anal. 101 (1988) 209-260.

[21] J. Warga, Optimal control of differential and functional equations. Academic Press, New York (1972).

[22] L.C. Young, Lectures on the calculus of variations and optimal control theory. Chelsea, New York (1980). 